\title{
Dead-space or dead-end: Can prolonged mechanical ventilation be predicted in all children after unifocalization?
}

\author{
Tyson A. Fricke, MBBS, BMedSci, ${ }^{\text {a,b,c }}$ and Igor E. Konstantinov, MD, PhD, FRACS ${ }^{\mathrm{a}, \mathrm{b}, \mathrm{c}, \mathrm{d}}$
}

\footnotetext{
From the a Department of Cardiac Surgery, The Royal Children's Hospital, Victoria, Australia, ${ }^{b}$ Department of Paediatrics, The University of Melbourne, 'Murdoch Children's Research Institute, Melbourne, Australia; and $\mathrm{d}$ Melbourne Children's Centre for Cardiovascular Genomics and Regenerative Medicine, Melbourne, Australia

Disclosures: Authors have nothing to disclose with regard to commercial support

Received for publication May 11, 2018; revisions received May 11, 2018; accepted for publication May 11, 2018; available ahead of print July 3, 2018.

Address for reprints: Igor E. Konstantinov, MD, PhD, FRACS, Royal Children's Hospital, Flemington Rd, Parkville, Victoria 3029, Australia (E-mail: igor.konstantinov@ @rch.org.au).

J Thorac Cardiovasc Surg 2018;156:1188-9

$0022-5223 / \$ 36.00$

Crown Copyright $@ 2018$ Published by Elsevier Inc. on behalf of The American Association for Thoracic Surgery https://doi.org/10.1016/j.jtcvs.2018.05.033
}

An interesting study by Koth and colleagues ${ }^{1}$ investigating the association between increased dead-space ventilation fraction $(\mathrm{Vd} / \mathrm{Vt})$ and prolonged mechanical ventilation in children undergoing surgery for tetralogy of Fallot, pulmonary atresia, and major aortopulmonary collaterals is published in this issue of The Journal of Thoracic and Cardiovascular Surgery. The study included 266 children who underwent unifocalization with complete repair and those who underwent unifocalization to a central shunt. The group has previously published the selection algorithm to determine if complete repair or shunting should be performed after unifocalization. ${ }^{2}$ Complete repair with ventricular septal defect (VSD) closure was performed if pulmonary artery pressure remained $<25 \mathrm{~mm} \mathrm{Hg}$ at full flow via a separate perfusion circuit while still on full cardiopulmonary bypass, a right ventricle/left ventricle pressure ratio $<0.5$ can be expected after VSD closure. ${ }^{2}$ If these criteria were not met the patients were shunted after unifocalization. Koth and colleagues concluded that an "elevated postoperative $\mathrm{Vd} / \mathrm{Vt}$ is associated with prolonged ventilation in patients with tetralogy of Fallot/pulmonary atresia/major aortopulmonary collaterals after unifocalization." 1 To fully understand the study and its implications, one must delve into the basic science on which the current study is based.

In physiology, dead-space refers to the part of the tidal volume of air that does not take part in gas exchange in the lungs. Either this is because air remains in the airways where gas exchange does not occur or air reaches alveoli in lungs that are not adequately perfused. It was Danish physician Christian Bohr (1855-1911) who first described the equation of dead-space relative to tidal volume in 1891. ${ }^{3}$ Bohr proposed that the difference between mean alveolar $\left(\mathrm{PACO}_{2}\right)$ and mixed expired $\left(\mathrm{PeCO}_{2}\right)$ partial pressures of $\mathrm{CO}_{2}$ can be used to determine the proportion

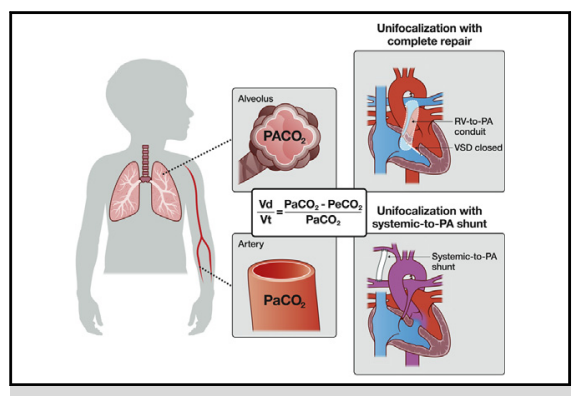

Bohr's formula might miscalculate dead-space ventilation fraction in patients with right-to-left shunting.

Central Message

Dead-space ventilation fraction might predict prolonged ventilation after unifocalization in pulmonary atresia, although this might not be applicable to patients with right-to-left shunting.

See Article page 1181. of the dead space. The equation was modified by Enghoff ${ }^{4}$ in 1938 by substituting $\mathrm{PACO}_{2}$, which was estimated using volumetric capnography in the Bohr equation, for arterial blood partial pressure of $\mathrm{CO}_{2}\left(\mathrm{PaCO}_{2}\right)$, from arterial blood gas sampling, to produce the formula applied by the authors:

$$
\frac{V d}{V t}=\frac{\mathrm{PaCO}_{2}-\mathrm{PeCO}_{2}}{\mathrm{PaCO}_{2}}
$$

When high concentrations of $\mathrm{CO}_{2}$ from the venous blood reach the arterial side through intracardiac rightto-left shunting, $\mathrm{PaCO}_{2}$ increases above $\mathrm{PACO}_{2}$ level. This would make $\mathrm{PaCO}_{2}$ inappropriate for calculation of dead space volume in children with intracardiac right-to-left blood shunting. Such intracardiac right-toleft shunting occurs in all patients with pulmonary atresia, who could not achieve complete repair with VSD closure, but instead had systemic-to-pulmonary shunt after unifocalization (Figure 1). Furthermore, even corrections of Enghoff's dead space formula for shunt effects still overestimate Bohr's dead space. ${ }^{5}$ The authors acknowledged this fact as a limitation of their study. Thus, it is not surprising that the group of patients with elevated $\mathrm{Vd} / \mathrm{Vt}$ had a higher proportion of patients with postoperative shunt physiology $(28 \%$ vs $14 \%$; $P=.001)$ compared with those with normal $\mathrm{Vd} / \mathrm{Vt}$. 


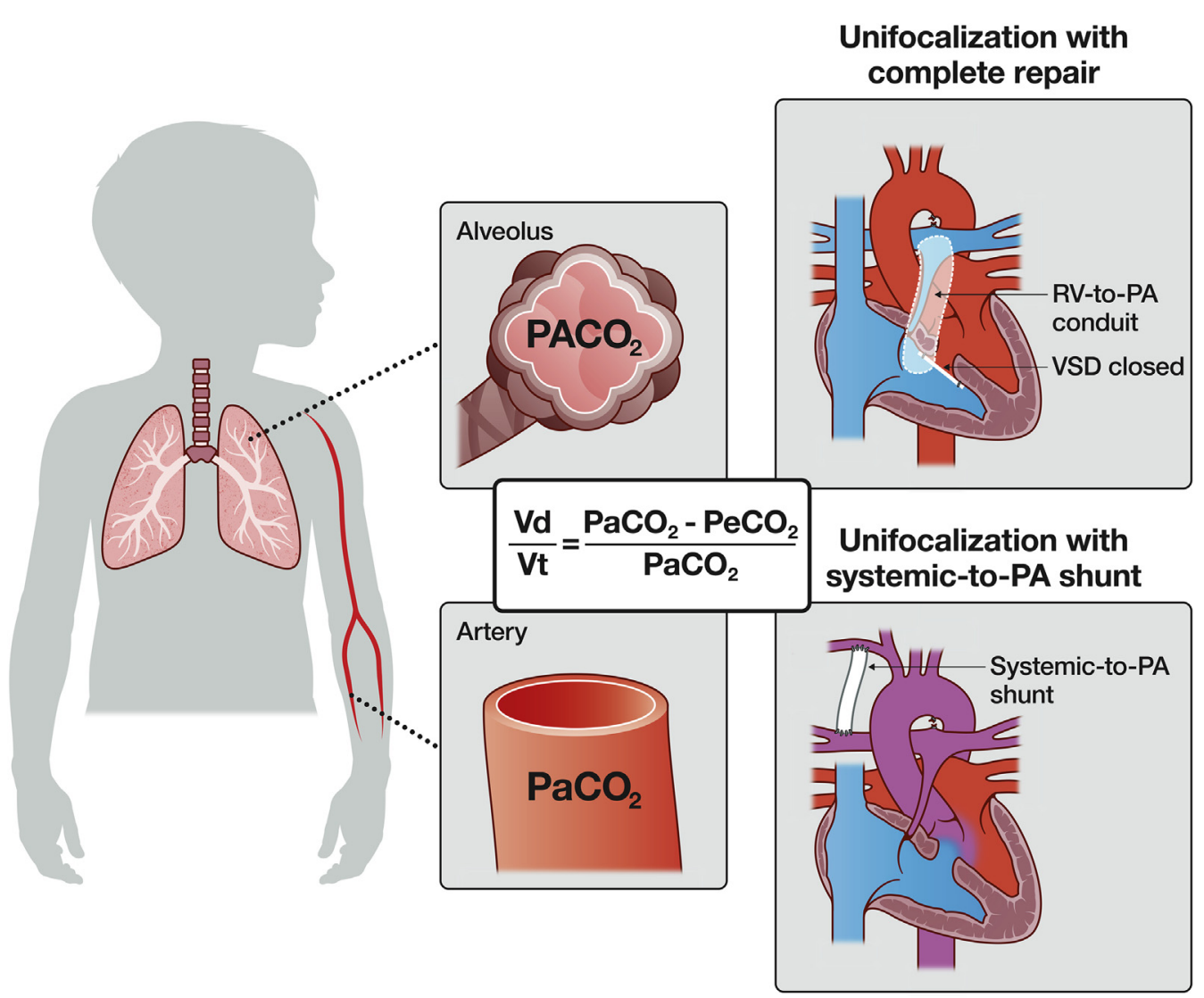

FIGURE 1. Enghoff's modification of Bohr's formula replaced alveolar partial $\mathrm{CO}_{2}$ pressure $\left(\mathrm{PACO}_{2}\right)$ by arterial partial $\mathrm{CO}_{2}$ pressure $(\mathrm{PaCO})$ to calculate ratio between dead-space volume $(V d)$ and tidal volume $(V t)$. Although this formula is appropriate for patients who underwent unifocalization with complete repair, it might yield the wrong dead-space ventilation fraction $(V d / V t)$ in those who underwent unifocalization without complete repair and were left with the right-to-left intracardiac shunt. Vd/Vt might predict prolonged ventilation after unifocalization in pulmonary atresia, although this might not be applicable to patients with right-to-left shunting. $R V$, Right ventricle; $P A$, pulmonary artery; $V S D$, ventricular septal defect.

In the analysis of all patients who had unifocalization, including both subgroups of patients (ie, complete repair and palliation with shunting), they showed that patients with an increased $\mathrm{Vd} / \mathrm{Vt}$ were at a higher risk of prolonged ventilation compared with those with a normal $\mathrm{Vd} / \mathrm{Vt}$. An elevated $\mathrm{Vd} / \mathrm{Vt}$ was not a risk factor for prolonged ventilation in multivariable analysis in either subgroup alone.

Although the results of this study should be interpreted with caution, this study is an important step forward. Identifying a noninvasive tool to reliably predict the risk for prolonged mechanical ventilation after unifocalization would be of great benefit to clinicians.

\section{References}

1. Koth AM, Kwiatkowski DM, Lim TR, Bauser-Heaton H, Asija R, McElhinney DB, et al. Association of dead space ventilation and prolonged ventilation after repair of tetralogy of Fallot with pulmonary atresia. J Thorac Cardiovasc Surg. 2018;156:1181-7.

2. Malhotra SP, Hanley FL. Surgical management of pulmonary atresia with ventricular septal defect and major aortopulmonary collaterals: a protocol-based approach. Semin Thorac Cardiovasc Surg. 2009;12:145-51.

3. Bohr C. Über die Lungenatmung. Skandinavisches Archiv Für Physiologie 2. 1891:236-238.

4. Enghoff H. Volumen inefficax. Bemerkungen zur Frage des schädlichen Raumes. Uppsala Läkareforen Forhandl. 1938;44:191-218.

5. Suarez-Sipmann F, Santos A, Böhm SH, Borges JB, Hedenstierna G, Tusman G. Corrections of Enghoff's dead space formula for shunt effects still overestimate Bohr's dead space. Respir Physiol Neurobiol. 2013;189:99-105. 